\title{
In-orbit verification, calibration and performance of the Heliospheric Imager on the STEREO mission
}

\author{
Chris Eyles $^{\mathrm{a}, \mathrm{b}, \mathrm{c}^{*}}$, Chris Davis $^{\mathrm{a}}$, Richard Harrison ${ }^{\mathrm{a}}$, Nick Waltham ${ }^{\mathrm{a}}$, Jean-Philippe Halain ${ }^{\mathrm{d}}$, \\ Emmanuel Mazy ${ }^{\mathrm{d}}$, Jean-Marc Defise ${ }^{\mathrm{d}}$, Russ Howard ${ }^{\mathrm{e}}$, Dan Moses ${ }^{\mathrm{e}}$, Jeff Newmark ${ }^{\mathrm{e}}$, Simon Plunkett ${ }^{\mathrm{e}}$ \\ ${ }^{\text {a }}$ Rutherford Appleton Laboratory, Chilton, Didcot, Oxfordshire OX11 0QX, UK; \\ ${ }^{\mathrm{b}}$ Grupo de Astronomía y Ciencias del Espacio, ICMUV, Universidad de Valencia, Spain; \\ 'School of Physics and Astronomy, University of Birmingham, Birmingham B15 2TT, UK; \\ ${ }^{\mathrm{d}}$ Centre Spatial de Liège, Université de Liège, ave Pré Aily B-4031 Angleur, Belgium; \\ ${ }^{\mathrm{e}}$ Space Science Division, Naval Research Laboratory, Washington DC, USA
}

\begin{abstract}
The Heliospheric Imager (HI) forms part of the SECCHI suite of instruments aboard the two NASA STEREO spacecraft which were launched successfully from Cape Canaveral AFB on 25 Oct 2006 (26 Oct UTC). Following lunar swingby's on $15 \mathrm{Dec}$ and 21 Jan respectively, the two spacecraft were placed in heliocentric orbits at approximately $1 \mathrm{AU}-$ one leading and one lagging the Earth, with each spacecraft separating from the Earth by $22.5^{\circ}$ per year.

Each HI instrument comprises two wide-angle optical cameras - HI-1 and HI-2 have $20^{\circ}$ and $70^{\circ}$ fields-of-view which are off-pointed from the Sun direction by $14.0^{\circ}$ and $53.7^{\circ}$ respectively, with the optical axes pointed towards the ecliptic plane. In this way the cameras will for the first time provide stereographic images of the solar corona, and in particular of Coronal Mass Ejections (CMEs) as they propagate outwards through interplanetary space towards the Earth and beyond. The wide-field coverage of HI enables imaging of solar ejecta from 15 to about 330 solar radii whilst the other SECCHI instruments ( 2 coronagraphs and an EUV imager) provide coverage from the lower corona out to 15 solar radii.

This paper briefly reviews the design and performance requirements for the instrument. The various activation, checkout and calibration activities before and after opening the instrument's protective cover or door (instrument 'first-light') are then described and it is shown that the instrument has met the design requirements, including CCD and camera imaging performance, correction for shutterless operation of the cameras, straylight rejection and thermal requirements. It is demonstrated from observations of a CME event on 24-25 Jan 2007 that the instrument is capable of detecting CMEs at an intensity of $1 \%$ of the coronal background. Lessons learnt during the design, development and in-orbit operation of the instrument are discussed.
\end{abstract}

Keywords: Sun, heliosphere, coronagraph, coronal mass ejection, CME, straylight, baffles, CCD camera.

\section{INTRODUCTION}

The launch of the two NASA STEREO (Solar Terrestrial Relations Observatory) spacecraft from Cape Canaveral Air Force Base at 01:52 UT on 26 October 2007 heralded a new era in the study of solar physics and solar-terrestrial relations.

After initial phasing orbits around the Earth, followed by lunar swing-bys in December 2006 and January 2007, the two spacecraft were injected into heliocentric orbits at approximately $1 \mathrm{AU}$. The Ahead, or ' $\mathrm{A}$ ', spacecraft is at rather less than $1 \mathrm{AU}$ and leads the Earth, whilst the Behind, or 'B', spacecraft at rather more than 1 AU lags behind the Earth. Each spacecraft separates from the Earth at a rate of $22.5^{\circ}$ per year, as measured from the Sun.

*Correspondence: cje@star.sr.bham.ac.uk

Solar Physics and Space Weather Instrumentation II, edited by Silvano Fineschi, Rodney A. Viereck Proc. of SPIE Vol. 6689, 668907, (2007) · 0277-786X/07/\$18 · doi: 10.1117/12.732822 
The two spacecraft carry identical payloads comprising the SECCHI (Sun Earth Connection Coronal and Heliospheric Investigation) suite of remote-sensing imaging instruments, together with instrumentation for in-situ measurements of particles, plasma and fields.

SECCHI is described in detail in ref [1] but in summary comprises five telescopes which together image the solar corona from the solar disc to $1 \mathrm{AU}$ and beyond:

- An extreme ultraviolet imager (EUVI) which images the chromosphere and lower corona out to $1.7 \mathrm{R}_{0}$ (solar radii) in four emission lines between 17.1 and $30.4 \mathrm{~nm}$.

- A pair of traditional white-light Lyot coronagraphs, COR-1 and COR-2, covering the coronal regions 1.4-4 $\mathrm{R}_{\mathrm{o}}$ and $2.5-15 \mathrm{R}_{\mathrm{o}}$ respectively, with bandpass from 650 to $750 \mathrm{~nm}$.

- A Heliospheric Imager (HI) which consists of two wide-angle cameras (HI-1 and HI-2) housed within a novel baffling and straylight rejection system. The HI cameras are off-pointed from the direction of the Sun, with their optical axes pointed towards the ecliptic plane. Jointly they cover the region $15-330 \mathrm{R}_{\mathrm{o}}$ (see Table 1).

This combination of instruments, together with the mission profile, provides outstanding new opportunities for the study of Coronal Mass Ejections (CMEs) and solar-terrestrial relations including:

- The first stereographic observations of the solar disc, the solar corona and CMEs

- The first observations of CMEs from a vantage point away from near-Earth space

- The first opportunity to observe geo-effective CMEs along the Earth-Sun line and in a field-of-view which contains the Earth.

\section{THE HELIOSPHERIC IMAGER}

The detection of CMEs by means of Thomson-scattered visible light as they propagate from the outer corona through the heliosphere to distances $\sim 1 \mathrm{AU}$ had its origin in the observations made by the zodiacal light photometers aboard the Helios spacecraft, launched in 1974 [2]. The SMEI (Solar Mass Ejection Imager) instrument [3] launched aboard the Coriolis spacecraft in 2003 has demonstrated the ability to map essentially the entire sky using three cameras with fieldsof-view of $60^{\circ} \times 3^{\circ}$ which scan around the sky each orbit.

The SECCHI HI is a third-generation instrument taking advantage of the STEREO mission design, together with a new baffling and straylight rejection concept [4]. The HI instrument design has been described in detail previously in $[4,5,6]$ and test results have been reported in $[7,8]$. Here we present a summary of the salient requirements and design features.

Figure 1(a) shows the geometry of the HI camera fields-of-view, together with the K- and F-coronal background [9] and the typical CME intensity. It should be noted that in general the CME signal is $\sim 1 \%$ of the background. Figure 1(b) shows the design concept of the instrument and Table 1 lists the main performance requirements and specifications.

The following important features are noted :

- The forward baffle consists of five cascaded diffracting vanes which provide the primary rejection of direct sunlight. Rejection levels of better than $10^{-9}$ and $10^{-11}$ are achieved at the entrance apertures of HI-1 and HI-2, respectively.

- $\quad$ HI-2 is further protected by the internal baffle which is designed to attenuate straylight from other bright objects such as planets and the antenna boom of the SWAVES instrument aboard the spacecraft.

- Both cameras use $2 \mathrm{k}$ x 2k large-format science-grade CCDs (e2v technologies CCD42-40) with 13.5 $\mu$ pixels [10]. However, for routine observations the images are $2 \times 2$ binned on-board in the SECCHI Electronics Box (SEB). The $\mathrm{CCD}$ are passively cooled by radiators facing deep space.

- In order to achieve the required sensitivities, total integration times $\sim 20 \mathrm{~min}$ and $1 \mathrm{hr}$ are required. To prevent CCD saturation and accumulation of background due to cosmic ray hits, each image is made up of a sequence of individual camera exposures which are then 'scrubbed' to remove cosmic rays and summed on-board in the SEB.

The HI instruments on the individual spacecraft are referred to as HI-1A, HI-2A, etc. 

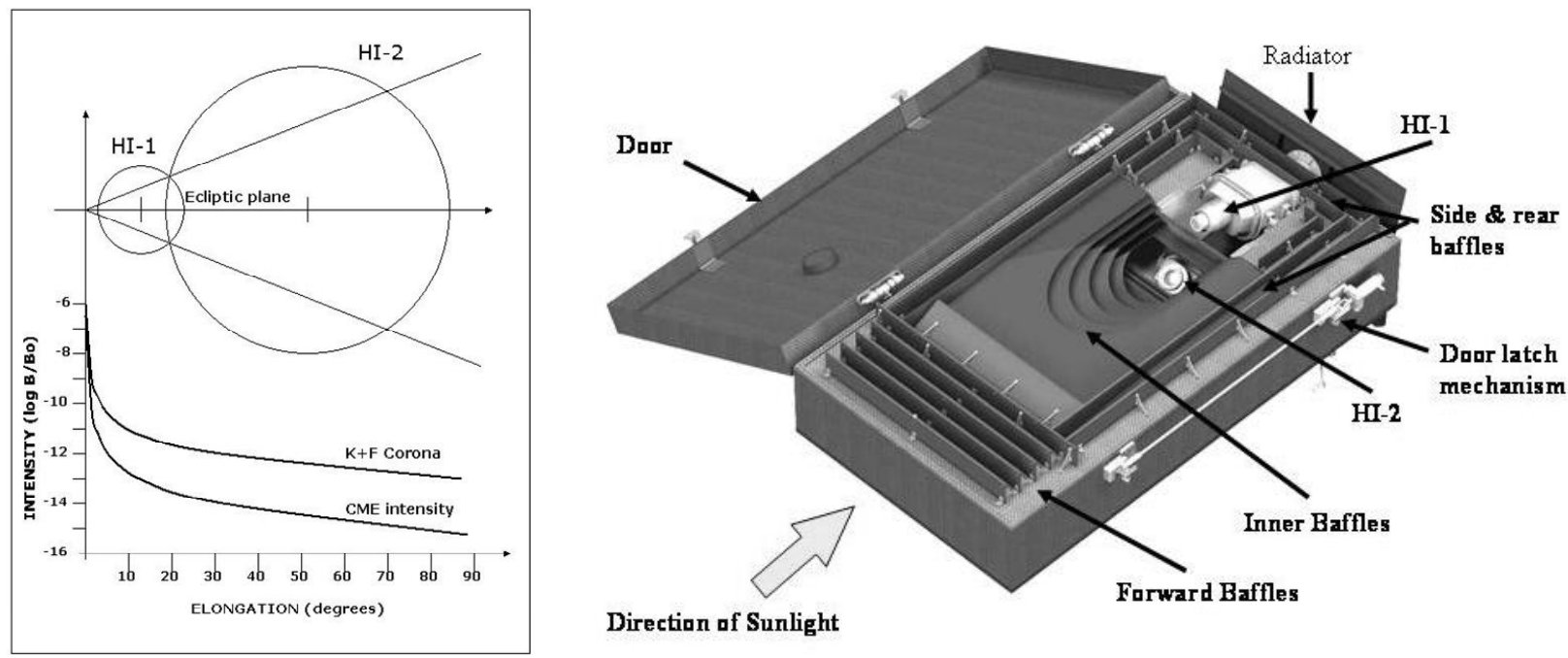

Figure 1. (a) HI fields-of-view and coronal intensity profiles based on a figure in reference [4], and (b) the HI design concept.

Table 1. Performance specifications of the HI instrument

\begin{tabular}{|l|c|c|}
\hline & $\mathrm{HI}-1$ & $\mathrm{HI}-2$ \\
\hline Direction of centre of FOV from Sun centre & $14.0^{\circ}$ & $53.7^{\circ}$ \\
\hline Angular field-of-view & $20^{\circ}$ & $70^{\circ}$ \\
\hline Angular range (in degrees) & $4-24^{\circ}$ & $18.7-88.7^{\circ}$ \\
\hline Angular range (in solar radii) & $15-90 \mathrm{R}_{0}$ & $70-330 \mathrm{R}_{0}$ \\
\hline CCD pixel size & $35^{\prime \prime}$ & $2^{\prime}$ \\
\hline Image array (2x2 binning) & $1024 \times 1024$ & $1024 \times 1024$ \\
\hline Image bin size & $70^{\prime \prime}$ & $4^{\prime}$ \\
\hline Spectral bandpass & $630-730 \mathrm{~nm}$ & $400-1000 \mathrm{~nm}$ \\
\hline Exposure time & $40 \mathrm{sec}$ & $50 \mathrm{sec}$ \\
\hline Exposure cadence & $1 \mathrm{~min}$ & $1 \mathrm{~min}$ \\
\hline Exposures per summed image sequence & 30 & 99 \\
\hline Summed image cadence & $40 \mathrm{~min}$ & $2 \mathrm{hr}$ \\
\hline Observing duty cycle & $50 \%$ & $69 \%$ \\
\hline
\end{tabular}
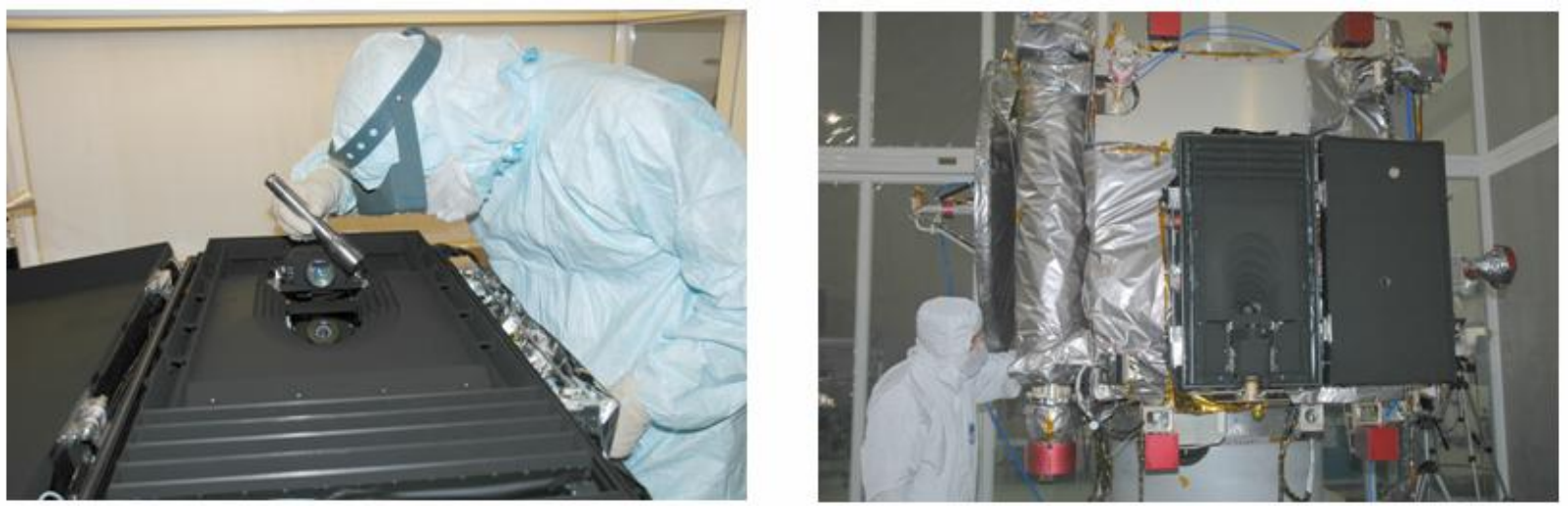

Figure 2. (a) HI-B undergoing final cleanliness inspection, and (b) HI-A mounted on the spacecraft at Astrotech, Florida. 


\section{MISSION OPERATIONS, INSTRUMENT CHECKOUT AND CALIBRATIONS}

\subsection{Initial mission phase}

The twin STEREO spacecraft were launched aboard a single Delta II rocket from Cape Canaveral Air Force Base at 01:52 UT on 26 October 2007 (8:52 pm EDT on 25 October).

A few hours after launch, the CCD decontamination heaters in all the SECCHI cameras on both spacecraft were switched on thereby ensuring that the CCDs were maintained at temperatures significantly above their surroundings, preventing the condensation of volatile outgassing material onto the CCD surfaces. The lens barrel heaters in the HI optics assemblies were also switched on. As a result the HI CCD temperatures were around $25-30^{\circ} \mathrm{C}$, the lens barrels were stabilized at $0^{\circ} \mathrm{C}$, whilst the $\mathrm{HI}$ structure temperatures were typically around $-20^{\circ} \mathrm{C}$ during this early phase of the mission.

A few days later, the HI Camera Electronic Boxes (CEBs) were switched on and dark images were taken with all cameras. Images were also taken using calibration LEDs which are mounted within each camera, providing optical stimulation of the CCDs by scattering off internal components within the focal plane assemblies. Although these images were dominated by dark charge due to the relatively high CCD temperatures, they did serve to confirm that all the cameras were fully functional.

The outgassing phase of the mission continued until 28 November when the decontamination heaters on both spacecraft were switched off. During this period checks of the functionality of all the SECCHI instruments continued.

When the decontamination heaters were switched off, the CCDs were cooled by the passive radiators over a timescale of $\sim 12$ hours. The final temperatures reached were around $-60^{\circ} \mathrm{C}$. It should be noted that at this stage the HI doors were still closed so these temperatures were significantly higher than those achieved after opening the doors (see Sect 4.2).

During the CCD cool-down, images were taken at periodic intervals to characterize the CCD dark charge variation as a function of temperature, thereby enabling the dark charge at the final operating temperatures achieved after opening the instrument doors to be estimated. The results obtained are described in Section 4.1.

Following the switch-off of the decontamination heaters it became possible to assess the CCD and camera performance with the CCDs at around $-60^{\circ} \mathrm{C}$. During this period the priority was to obtain dark calibration images for all cameras prior to opening the instrument doors. Since the cameras do not have shutters and the door deployment mechanisms are 'one shot' it is not possible to take dark images after door deployment. In addition, calibration LED images were obtained. These will be used for determining the small spatial scale or pixel-to-pixel variations of CCD response for comparison with values obtained from sky data.

Although dark images were obtained from all cameras, in some cases these were compromised by 'light leaks' around the edges of the instrument door. These were particularly troublesome at certain spacecraft attitudes during the early phasing orbits when stray light from the nearby bright Earth caused significant background in some parts of the images. Although dark images have been acquired for the instrument calibration database we do not expect to depend significantly on them since the CCD dark charge at operating temperatures is so low (see Section 4.1) and the occurrence of 'hot pixels' at these temperatures is also very rare.

\subsection{HI door deployment and first lights}

The HI-A door deployment was performed on 13 December 2006, two days before the STEREO-A lunar swing-by and injection into heliocentric orbit. The outstanding quality of the images was immediately apparent - see Figure 3 . In addition to the F-corona, the Milky Way, several clusters and numerous stars are all visible.

The HI-1A image is $20^{\circ}$ across and the Sun centre is $4^{\circ}$ off the right-hand edge. The F-coronal intensity at $4^{\circ}$ is $\sim 10^{-10} \mathrm{~B}_{0}$ $\left(\mathrm{B}_{0}\right.$ is the direct surface brightness of the Sun's disc) which in itself is a remarkable demonstration of the performance of the instrument. The bright object with the vertical streak is the saturated image of Venus. The vertical streak arises from two effects - (a) when the CCD pixels saturate as a result of the charge exceeding the full-well depth, the excess charge predominantly bleeds up and down the columns, and is prevented from spreading horizontally by the channel stops, and (b) the shutterless operation of the cameras results in a series of 'mini exposures' as the image is clocked down the CCD during the clear and readout operations, in addition to the main exposure, causing a vertical trailing of bright objects. 

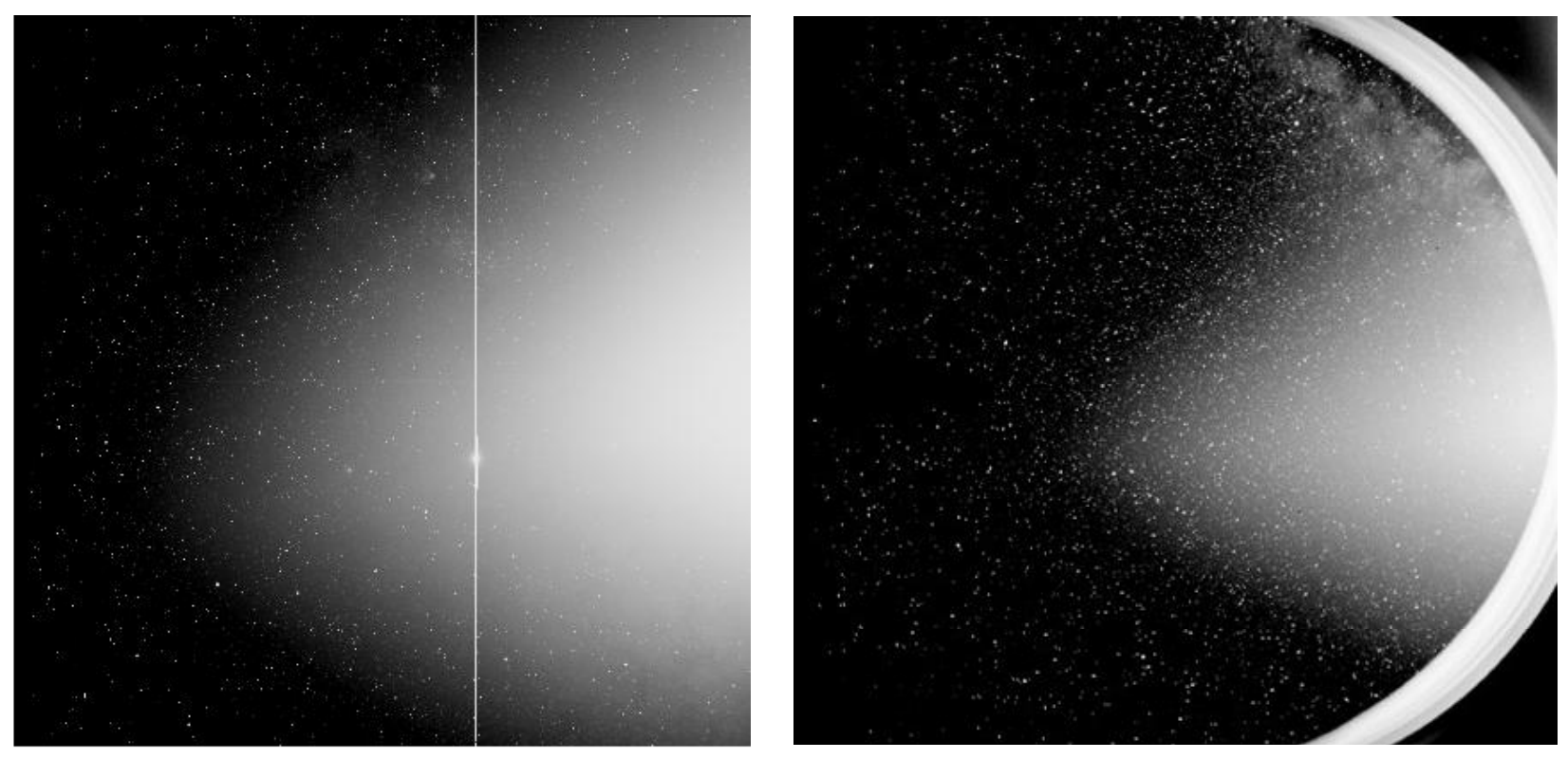

Figure 3. (a) HI-1A first light image, and (b) HI-2A first light from 13 December 2007.

The HI-2 image is $70^{\circ}$ across and the Sun is $18.7^{\circ}$ off the right-hand edge. Here we are seeing the F-coronal intensity at $10^{-12} \mathrm{~B}_{0}$, although the coronal profile can be followed right across the field-of-view. The bright semicircular rings on the right hand side are images of the internal baffle edges, illuminated primarily by straylight from the Earth.

HI-B door deployment and first light occurred on 11 January 2007, and resulted in spectacular images of Comet McNaught [11,12]. STEREO-B final lunar swing-by and heliocentric insertion was on 21 January.

\subsection{HI commissioning}

After deployment of the doors, the temperature of the entire instrument dropped fairly rapidly on a timescale $\sim 12 \mathrm{hr}$. Adjustments were made to the lens barrel heater set points. CCD temperatures around $-80^{\circ} \mathrm{C}$ were achieved on all cameras, giving very low dark charge and high tolerance to radiation damage effects. Details are given in Section 4.2.

The optical imaging performance was assessed, in particular the widths of the point source response functions (PSFs) of the telescopes, which were found to be generally similar to pre-launch values. Details are given in Section 4.3.

An issue specific to HI-B was that soon after first light, the Earth entered and rapidly crossed the HI-2B field-of-view between 24 January and 8 February causing substantial CCD saturation. The Earth then moved across the HI-1B field towards the Sun before reversing its apparent motion, re-entering HI-2B and eventually leaving HI-1B in early-April. Although the brightness of the Earth was decreasing over this period, it continued to cause significant local saturation and image ghosts. An example of the bright Earth effects in HI-2B from early February is discussed in Section 4.4.

It must be emphasized that the bright Earth effects in HI-B do not represent an instrumental 'problem' - they are an inevitable feature of the mission design since the ' $\mathrm{B}$ ' spacecraft is moving out away from the Sun and behind the Earth during this early mission phase. In fact the images demonstrate the remarkable dynamic range of the cameras.

\subsection{HI calibrations}

The following HI calibrations were performed during commissioning:

- Straylight calibration off-points

The spacecraft was off-pointed from nominal pointing in a series of steps in HI pitch up ranging from $1.5^{\circ}$ towards the Sun to $1^{\circ}$ away from the Sun. This enabled the straylight rejection of the forward baffle to be verified. 
- Spacecraft $180^{\circ}$ rolls and $90 / 270^{\circ}$ simultaneous rolls

In these each spacecraft was independently rolled through $180^{\circ}$ so that both sets of $\mathrm{HI}$ cameras were viewing the same region of sky in the ecliptic plane. The spacecraft were also simultaneously rolled through $90^{\circ}$ and $270^{\circ}$ so that the cameras viewed the same region of sky perpendicular to the ecliptic. Each case allows comparison between the response of HI-A and HI-B cameras.

- Calibration LED + sky background image pairs

Since the HI cameras do not have shutters, it is not possible to take dark images or images just using the calibration LEDs. Instead an image is taken with the LEDs stimulated and then a second sky image is taken immediately afterwards using the same exposure time. The difference between the two represents the LED signal.

- Exposure time linearity sequences

A series of images is taken using eight exposure times ranging from 0 to $110 \mathrm{sec}$.

The calibration LED and exposure time linearity sequences are being repeated at roughly 3-monthly intervals, in order to monitor the response of the cameras throughout the mission. The straylight off-points and $90 / 270^{\circ}$ roll calibrations may also be repeated at infrequent intervals (e.g. every 6 months).

\subsection{HI science operations}

Science operations are relatively simple for HI since the instrument performs a continuous synoptic, or monitoring, observing programme. The details of the programme were refined during the commissioning phase and the final values arrived at for the exposure times, cadences, etc are given in Table 1. The factors taken into account were:

- Remain within the camera dynamic range at the brightest regions of the F-corona, with some margin; this limited the exposure time for HI-1 to $40 \mathrm{sec}$.

- Number of saturated stars typically no greater than $\sim 6$; this limited the HI-2 exposure time to $50 \mathrm{sec}$.

- Achieve a fairly high observing efficiency (total exposure time as fraction of elapsed time), particularly for HI-2.

- Take account of overall constraints on SEB image processing load and need to take exposures at multiples of $1 \mathrm{~min}$ interval to ease exposure scheduling.

A total of 36 summed images ( $2 \times 2$ binned) for HI- 1 and 12 for HI- 2 are taken every $24 \mathrm{hr}$. In addition, one highresolution (unbinned) image is normally taken for each telescope every $24 \mathrm{hr}$ for performance monitoring.

Momentum dumps are performed at approximately 6-weekly intervals on each spacecraft in order to offload the reaction wheels in the attitude control system. This operation interrupts normal observations and these occurrences are being used to alternately repeat either the exposure time linearity sequence, or the LED + sky background image pairs.

\section{INSTRUMENT CHECKOUT AND CALIBRATION RESULTS}

\subsection{CCD cooling and dark charge measurements}

An example of the cooling curves and the variation of dark charge with temperature when the decontamination heaters were switched off is given in Figure 4 for HI-1A. The results for the other telescopes were essentially similar.

The variation of dark charge for a non-inverted-mode CCD can be characterized by the relationship -

$$
\mathrm{I}_{\text {dark }}=\mathrm{A} \mathrm{T}^{-3} \exp (-6400 / \mathrm{T})
$$

where $\mathrm{T}$ is the temperature in $\mathrm{K}[10]$. 

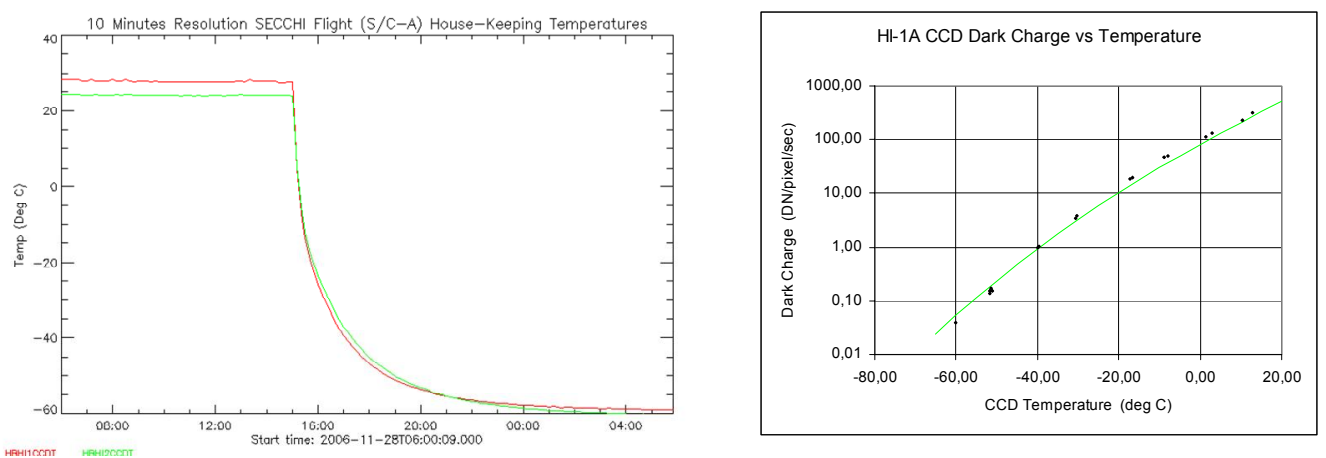

Figure 4. (a) Cooling curves for HI-1A and HI-2A CCDs, and (b) dark charge versus temperature.

The above relationship was fitted to the measured dark charge values and the resultant best fit is shown in Figure 4(b). It can be seen that quite a good fit is achieved despite the wide range of temperatures. Similar fits were obtained for the other cameras. The fitted values for A were then used to predict the expected dark charge at the typical CCD operating temperatures of approximately $-80^{\circ} \mathrm{C}$.

The predicted values for dark charge at $-80^{\circ} \mathrm{C}$ are given in Table 2 , together with the values at $+20^{\circ} \mathrm{C}$. The dark charge is measured in DN (digital number), i.e. CEB analog-to-digital converter units. (For the 14-bit ADC used in the CEB the maximum value is 16383 .) The nominal dark charge at $+20^{\circ} \mathrm{C}$ as quoted by the CCD manufacturer, e $2 \mathrm{v}$ technologies Ltd, is shown for comparison, the value shown being based on the manufacturer's value of $20000 \mathrm{e}^{-} / \mathrm{pixel} / \mathrm{sec}$, together with a conversion factor of $1 \mathrm{DN}=15 \mathrm{e}^{-}$for the SECCHI CEBs.

Table 2. Predicted CCD dark charge values at $-80^{\circ} \mathrm{C}$

\begin{tabular}{|c|c|c|c|c|c|}
\hline \multirow{2}{*}{$\begin{array}{c}\mathrm{CCD} \\
\text { Temperature }\end{array}$} & \multicolumn{5}{|c|}{ Dark charge (DN/pixel/sec) } \\
\cline { 2 - 6 } & HI-1A & HI-2A & HI-1B & HI-2B & e2v value \\
\hline$+20^{\circ} \mathrm{C}$ & 505.7 & 392.0 & 496.5 & 402.0 & 1300 \\
\hline$-80^{\circ} \mathrm{C}$ & 0.0018 & 0.0014 & 0.0017 & 0.0014 & - \\
\hline
\end{tabular}

It can be seen that the actual dark charge of the devices used in HI is substantially below the manufacturer's quoted nominal value. Furthermore, the predicted dark charge at the typical operating temperatures of $-80^{\circ} \mathrm{C}$ achieved after opening the HI doors is completely negligible. Taking a typical sequence of 99 summed 50 sec exposures for HI- 2 we arrive at $\sim 30 \mathrm{DN} /$ bin allowing for $2 \times 2$ binning. Even in the darkest regions of a summed HI-2 image the sky background signal is three orders of magnitude larger at $\sim 30,000 \mathrm{DN} / \mathrm{bin}$ for the summed image. This is an excellent result demonstrating that there is a large margin for possible degradation of CCD performance due to radiation damage.

\subsection{Instrument thermal status after door deployment}

Table 3 summarises the thermal status of the instrument at equilibrium following door deployment. As expected from the thermal design and modeling the instrument runs very cold, with structure temperatures well below zero. However, all temperatures are well within operational limits, with the exception of HI-1B lens barrel. Due to a 'thermal leak' HI-1B cannot be controlled at $-15^{\circ} \mathrm{C}$ and the selected set-point of $-29^{\circ} \mathrm{C}$ is only just within the range of the temperature monitor. This problem was known before launch and is of no consequence - the optics performance is in no way compromised by the low temperature. All CCD temperatures are close to the design value of $-80^{\circ} \mathrm{C}$.

\subsection{Imaging performance}

Initial results from fitting to star fields indicate that the plate-scales, distortion parameters, etc are all consistent with prelaunch values. Table 4 gives the measured widths of the point source response function. 
Table 3. Instrumental thermal status after door deployment.

\begin{tabular}{|l|c|c|c|}
\hline & Operational Limits & HI-A & HI-B \\
\hline Structure & $-120^{\circ} \mathrm{C}$ to $+100^{\circ} \mathrm{C}$ & $-70^{\circ} \mathrm{C}$ to $-23^{\circ} \mathrm{C}$ & $-68^{\circ} \mathrm{C}$ to $-29^{\circ} \mathrm{C}$ \\
\hline S/C Interface & $-15^{\circ} \mathrm{C}$ to $+45^{\circ} \mathrm{C}$ & $-1^{\circ} \mathrm{C}$ & $0^{\circ} \mathrm{C}$ \\
\hline HI-1 CCD & $-100^{\circ} \mathrm{C}$ to $-70^{\circ} \mathrm{C}$ & $-83^{\circ} \mathrm{C}$ & $-80^{\circ} \mathrm{C}$ \\
\hline HI-2 CCD & $-100^{\circ} \mathrm{C}$ to $-70^{\circ} \mathrm{C}$ & $-80^{\circ} \mathrm{C}$ & $-76^{\circ} \mathrm{C}$ \\
\hline HI-1 Lens Barrel & $-30^{\circ} \mathrm{C}$ to $+30^{\circ} \mathrm{C}$ & $-15^{\circ} \mathrm{C}$ & $-29^{\circ} \mathrm{C}$ \\
\hline HI-2 Lens Barrel & $-30^{\circ} \mathrm{C}$ to $+30^{\circ} \mathrm{C}$ & $-15^{\circ} \mathrm{C}$ & $-15^{\circ} \mathrm{C}$ \\
\hline CEB Internal & $-35^{\circ} \mathrm{C}$ to $-50^{\circ} \mathrm{C}$ & $-13^{\circ} \mathrm{C}$ & $-13^{\circ} \mathrm{C}$ \\
\hline
\end{tabular}

* Controlled to set-point by SEB under closed-loop control

Table 4. Pre-launch and on-orbit values for width of optics point source response function (on axis).

\begin{tabular}{|l|c|c|c|}
\hline \multirow{2}{*}{} & \multicolumn{2}{|c|}{ Pre-Launch PSF (pixels) } & On-Orbit PSF (pixels) \\
\cline { 2 - 4 } & FWHM & HEW & FWHM estimated \\
\hline HI-1A & $3-4$ & $3-4$ & $\sim 3-4$ \\
\hline HI-2A & $3-6$ & $3-6$ & $\sim 3-8$ \\
\hline HI-1B & $3-4$ & $3-4$ & $\sim 3-4$ \\
\hline HI-2B & $3-10$ & $3-12$ & $\sim 3-12$ \\
\hline
\end{tabular}

The relatively poor imaging quality of HI-2B was known about before launch. Optical tests during spacecraft integration showed that it is not a simple focus setting error. The problem does not have any significant consequences for the prime science objective of CME imaging - CMEs are relatively diffuse structures - although it does make procedures for star subtraction and data analysis for stellar photometry studies, etc more complex. Work is underway to model the PSFs.

\subsection{Effects of bright Earth in HI-B}

Figure 5 shows the effects caused by the bright Earth in HI-2B on 6 February 2007. It should be emphasized that this is an extreme example when the Earth was first crossing HI-2B and the spacecraft-Earth separation was still only $\sim 0.007$ AU. The apparent brightness of the Earth is continually decreasing with increasing separation throughout the mission.
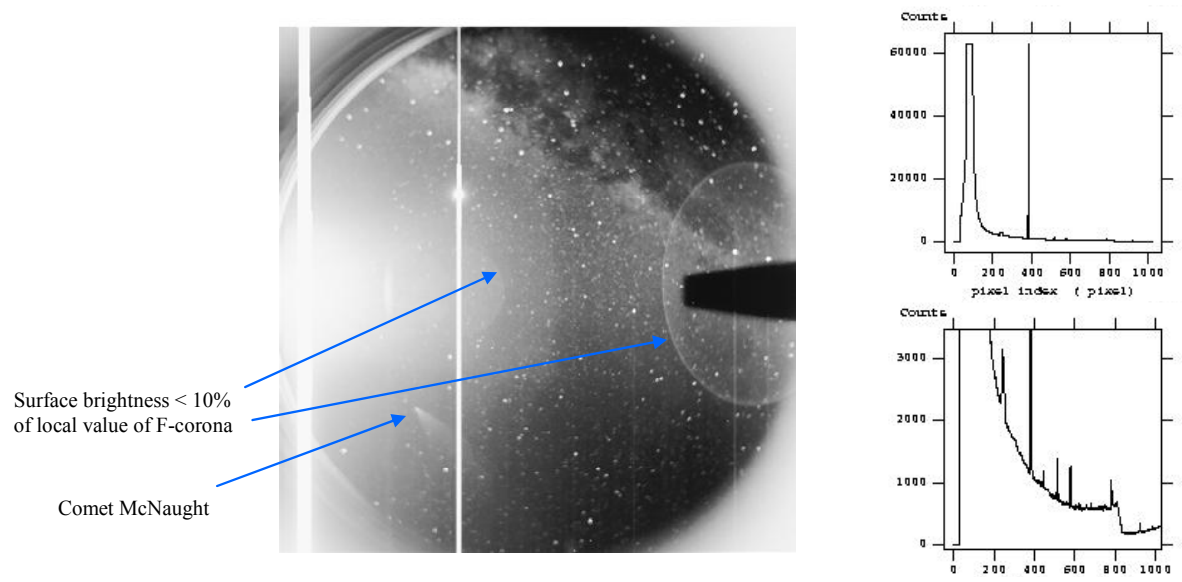

Figure 5. (a) HI-2B $10 \mathrm{sec}$ exposure on 6 February showing effects of bright Earth, and (b) profiles through centre of the image.

There is serious saturation of the CCD and local glare caused by the Earth near the left-hand edge of the image, and to a lesser extent by the Moon closer to the centre, but it is confined to vertical columns in these regions. The total signal 
from the Earth in the $10 \mathrm{sec}$ exposure is estimated at $\sim 5 \times 10^{10}$ photoelectrons per pixel. This is a factor $\sim 2.5 \times 10^{5}$ larger than the CCD full-well depth, demonstrating the remarkable dynamic range of the camera.

Over much of the image there is very little degradation - the F-coronal profile, Comet McNaught and the Milky Way are all still clearly visible. The straylight in the corners of the image is due to light from the Earth scattering from the lens barrel internal surfaces. Ghost images from the optics are clearly visible although the profiles through the centre of the image show that even the brightest features in the ghosts are $<10 \%$ of the local intensity of the F-corona.

\subsection{Straylight off-point calibration results}

A straylight off-point calibration was performed on HI-A on 30 January. Exposures were taken at spacecraft off-point angles of $0.25^{\circ}, 0.5^{\circ}, 0.75^{\circ}, 1.0^{\circ}$ and $1.5^{\circ}$ towards the Sun, and $0.5^{\circ}$ and $1.0^{\circ}$ away from the Sun in HI pitch, relative to nominal pointing. Visual inspection of the images indicated very little change except that there was clearly a substantial amount of straylight in both HI-1 and HI-2 images at the $1.5^{\circ}$ off-point.

For each off-point, the F-coronal intensity was measured at eight locations across the centerline of the field of view. The values obtained are plotted against distance from the Sun centre (allowing for the off-point angle) in Figure 6(a) and (c). A power-law profile $\mathrm{R}^{-\mathrm{n}}$ was fitted to the data points corresponding to nominal pointing, and Figures (b) and (d) show the residuals after the fitted profile is subtracted from the data points.

If the straylight intensity does not change with off-point angle then all the data points should lie on the same curve. This is evidently the case for all off-points except for $-1.0^{\circ}$ and to a much smaller extent $-0.75^{\circ}$, where the measured values lie significantly above the fitted profiles.
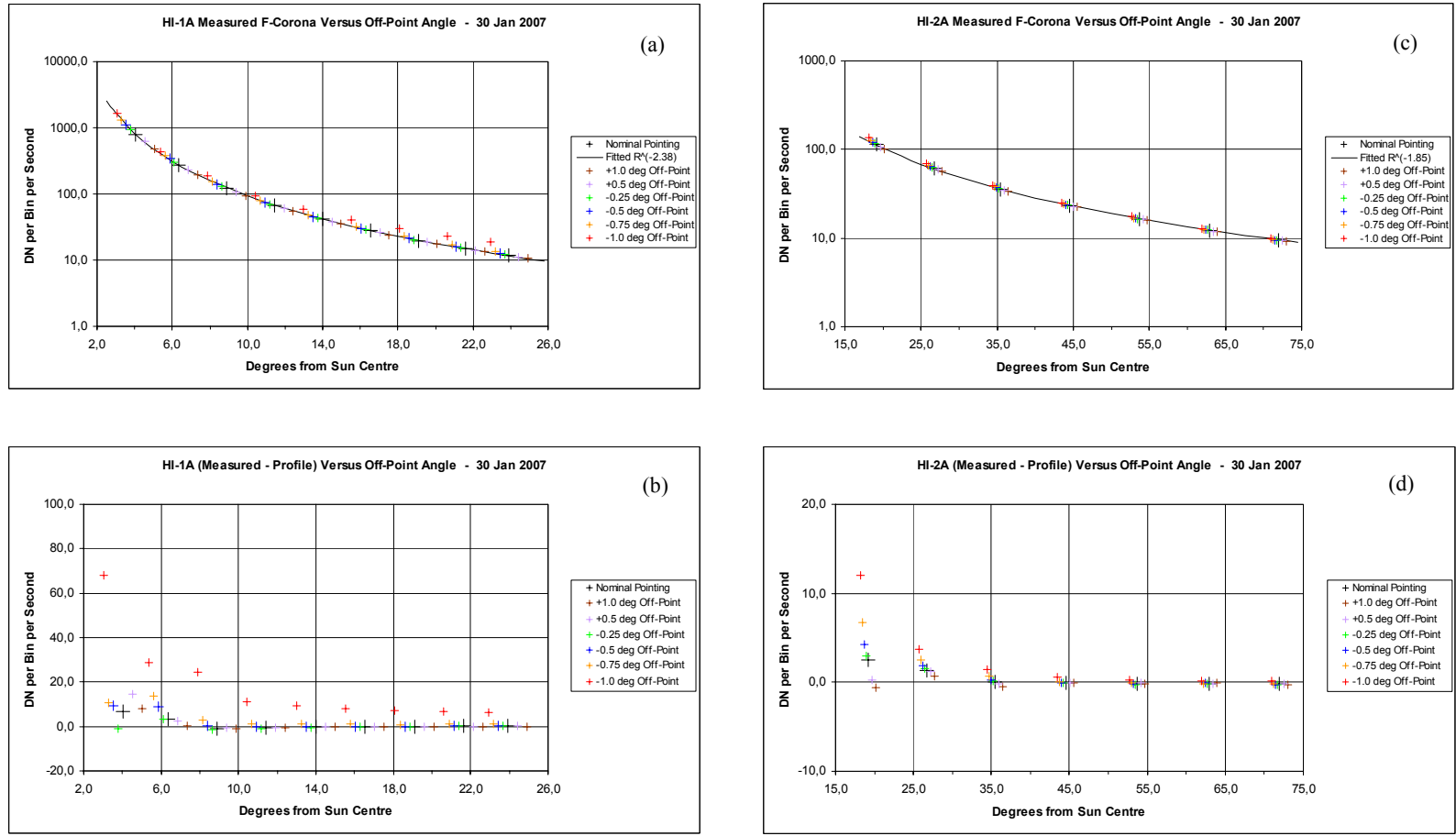

Figure 6. (a) Measured F-coronal intensity plotted against angle from Sun centre for HI-1A, and (b) residual intensity after subtracting fitted profile; (c) and (d) are same plots for HI-2A.

The following conclusions are drawn:

- There is at least $0.5^{\circ}$ margin in the pointing alignment of HI-1A relative to the Sun direction.

- In the nominal case, the straylight level in HI-1A is less than $\sim 2-3 \%$ of the F-coronal background across the entire field-of-view; a similar limit applies to HI-2A. 
Results for HI-B were broadly similar except that the margin in the pointing alignment is a little smaller, $\sim 0.3^{\circ}$.

It is noted that the profiles for both HI-1 and HI-2 are well-fitted by a $\mathrm{R}^{-\mathrm{n}}$ power law, although the values for the power law index are very different. For HI-1A, the value of -2.38 is consistent with the model of Koutchmy and Lamy [9], who give values of -2.25 in the ecliptic and -2.47 towards the poles. The roll angle of spacecraft ' $A$ ' relative to the ecliptic on 30 January was $15.0^{\circ}$. However, for HI-2A the value is -1.85 . It is suggested that the reason for this difference may be the different spectral response of the two cameras.

It is clear that the $\mathrm{HI}$ is well placed for making definitive measurements of the F-coronal intensity, including variation with angle to the ecliptic, by taking advantage of different spacecraft roll angles throughout the early mission and in particular during roll calibrations of the SCIP instruments.

\section{AN EARLY CME RESULT}

One of the first CMEs to be observed by HI was an event seen in HI-1A between 14:00 UT on 24 January and 04:00 UT on 25 January 2007. The event was first observed by LASCO C2 and C3, and was followed through HI-1A out to about $50 \mathrm{R}_{\mathrm{o}}$ over a total period of $15 \mathrm{hr}$. Unfortunately, there was a data gap on HI-1A after 04:00 UT, but the event was detected subsequently by HI-2A and later by SMEI on 26-28 January.

At the time of the observations both STEREO spacecraft were still very close to the Earth, with a total separation angle at the Sun of just over $0.5^{\circ}$ and with viewing locations only just outside the Sun-Earth line. Consequently, the event was not observed by the HI-B instruments.

Detailed analyses of this event, including comparison with other instruments and brief descriptions of other early events observed by HI, will appear in a HI CME 'first light' paper [13]. Here we present the essential observations to demonstrate that $\mathrm{HI}$ is easily meeting its requirements in terms of CME detection and will be capable of making outstanding contributions to CME studies later in the mission when observations away from the Sun-Earth line become possible.

Figure 7 shows an image of the event from HI-1A early on 25 January, the image being the sum of twenty five exposures each of $24 \mathrm{sec}$ duration. The raw image was first corrected for the 'shutterless' operation of the camera and then the Fcoronal background was removed. The background was determined by taking the minimum intensity for each image pixel over a number of similar images around the same time, thereby measuring the underlying background upon which transient features (and the time-drifting images of stars) are superimposed.

The image is $20^{\circ}$ across and the Sun is $4^{\circ}$ off the right-hand edge. The quality of the image is quite outstanding with stars down to $12-13^{\mathrm{m}}$ visible. There is no evidence of 'trailing' of the images of the brighter stars, demonstrating the effectiveness of the shutterless correction. The bright objects in the lower part of the image are Venus (towards the bottom left) and Mercury, both of which cause local saturation of the CCD. The structure of the CME expanding away from the East limb of the Sun at around $600 \mathrm{~km} / \mathrm{sec}$ is clearly visible. The presence of Venus and Mercury in the FOV graphically demonstrates the potential for combining remote sensing observations with in-situ measurements.

In Figure 8(a) we plot the profile of the raw intensity before subtraction of the F-coronal background measured across the centerline of the HI field-of-view. The plot is actually for an earlier image during the event, at 22:01 UT on 24 January, but the essential features are the same. The profile is dominated by the F-coronal signal with small spikes due to stellar images.

Figure 8(b) shows a plot on the same intensity scale but with the coronal background removed. The peak of the CME front is clearly visible at $\sim 900$ intensity units and structure associated with the CME is clearly distinguishable down to 200 units, or less. It should be emphasized that this profile was taken across a line of single image pixels so represents a worst case. Since the structure in CMEs is relatively diffuse, features significantly fainter than this are visible.

Referring back to Figure 8(a) we can see that the corresponding F-coronal signal in this part of the image is $\sim 20,000$ units so that the peak intensity of the CME front is $\sim 4.5 \%$ of the coronal background. Crucially, we have demonstrated that we can readily detect CME features down to $1 \%$ or less of the F-coronal intensity. These measurements were made at about $40 \mathrm{R}_{\mathrm{o}}$. Figure $1(\mathrm{a})$ shows that at this radius we are detecting CMEs at a level of about $10^{-14} \mathrm{~B}_{0}$. 


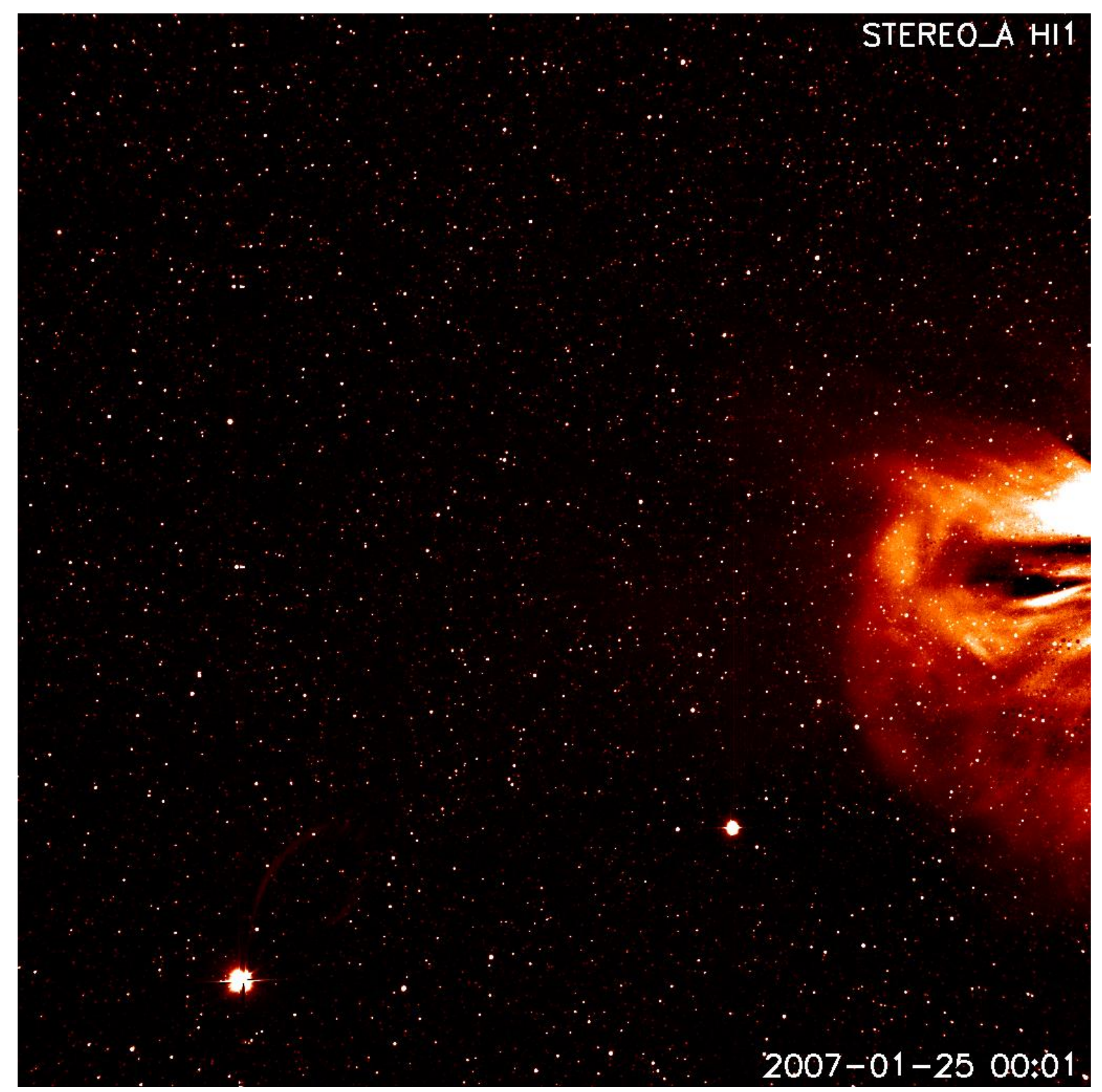

Figure 7. A HI-1A image showing the CME event on 24-25 January 2007.
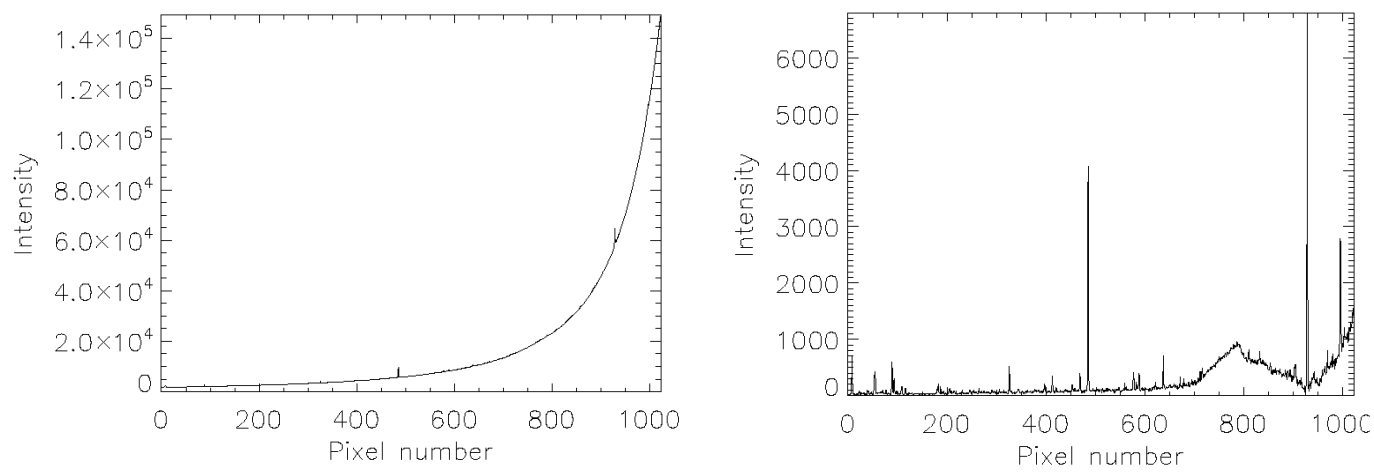

Figure 8. (a) Profile of raw intensity across the HI-1 field-of-view, and (b) the profile with F-coronal background removed. 
The same CME was subsequently detected by HI-2A, although it was fading rapidly as it moved across the field-of-view. The front was visible to around $30^{\circ}$ from the solar limb, although there appeared to be some 'forerunner' structures visible out to around $42^{\circ}[13]$.

\section{CONCLUSIONS}

The observations of the 24-25 January CME event clearly demonstrate that $\mathrm{HI}$ is meeting its performance requirements in terms of the capability to detect CMEs at the level of $1 \%$ or less of the F-coronal background, and that major breakthroughs can be expected as the mission progresses and viewing of the same regions along the Sun-Earth line by both sets of instruments becomes possible later this year.

The straylight calibration off-points demonstrate that the forward baffle system is rejecting the direct solar flux to a level nearly two orders of magnitude below the F-coronal background over the entire fields-of-view of HI-1 and HI-2.

In general the instruments are performing entirely to specifications in all important respects. The only respects in which the performance is not ideal all have very little impact on the ability to meet the prime science objectives:

- The thermal leak in HI-1B lens barrel is of no consequence. This problem was known about before launch.

- The degraded point-source response function of HI-2B optics has no impact on CME imaging, although it does make data analysis requirements for star subtraction and stellar photometry studies somewhat more complex. Again, this problem was known about before launch but schedule constraints prevented any remedial action being taken.

- There is some occasional instability in the apparent pointing attitude of HI-1B, causing star images to be slightly spread or smeared. The origin of this effect is at present unknown. The proportion of images significantly affected is small ( $\leq$ few \%).

- The bright Earth 'problem' in HI-B caused significant CCD saturation, straylight and ghosting problems in the early months of the science operations phase. However, by the time the optimum observing period for HI begins in late2007 , with spacecraft total separation angle of say $40^{\circ}$, the effects will be minor and similar to those from other bright planets, e.g. Venus.

The design and development of the $\mathrm{HI}$ instruments was extremely challenging, both technically and programmatically. Nevertheless it is difficult to suggest ways in which the 'end product' could be significant improved other than:

- Had mass and accommodation constraints allowed, an offset pointing mechanism would have permitted on-orbit adjustment of the instrument pitch angle relative to the Sun. In fact, the instrument was originally designed to have an offset angle for HI-1 of $13.65^{\circ}$, giving a small overlap between COR-2 and HI-1 fields-of-view which would have assisted the cross-calibration of the instruments. However, fairly late in the instrument development programme it was decided to adopt a conservative approach and introduce a further $20^{\prime}$ offset when mounting the instrument on the spacecraft, given the disastrous consequences of an alignment error in the wrong direction. The straylight calibration off-points show that this was unnecessary for HI-A, although HI-B does not have quite the same margin. An offset pointing mechanism would have avoided this.

- Shutters in the cameras would have avoided the complication of a shutterless correction in the data analysis software and would permit dark calibration images to be taken throughout the mission. However, the correction is in fact very effective as is apparent from the quality of the image in Figure 7.

- The response to the bright Earth and the straylight rejection requirements highlight the need for extreme cleanliness when building coronagraphs of this type, together with the need minimize ghost images in the optics, optimization of AR coatings, etc.

- An attempt was made to reduce the impact of the bright Earth by placing an occulter in front of the HI-2 CCD. However, it was only when detailed mission predictions became available late in the development programme that it became apparent that the occulter would only be effective for a limited period during the mission. In fact it is difficult to see what could be done to avoid this problem which is an inevitable consequence of the STEREO mission concept. 


\section{ACKNOWLEDGEMENTS}

The Heliospheric Imager (HI) instrument was developed by a collaboration which included the University of Birmingham and the Rutherford Appleton Laboratory, both in the UK, and the Centre Spatial de Liège (CSL), Belgium, and the US Naval Research Laboratory (NRL), Washington DC, USA.

The STEREO/SECCHI project is an international consortium of the Naval Research Laboratory (USA), Lockheed Martin Solar and Astrophysics Lab (USA), NASA Goddard Space Flight Center (USA), Rutherford Appleton Laboratory (UK), University of Birmingham (UK), Max-Planck-Institut für Sonnensystemforschung (Germany), Centre Spatial de Liege (Belgium), Institut d'Optique Théorique et Appliquée (France), Institut d'Astrophysique Spatiale (France).

The USA institutions were funded by NASA; the UK institutions by Physics and Astronomy Research Council (PPARC); the German institutions by Deutsche Zentrum für Luft- und Raumfahrt e.V. (DLR); the Belgian institutions by Belgian Federal Science Policy Office; the French institutions by Centre National d'Etudes Spatiales (CNES) and the Centre National de la Recherche Scientifique (CNRS). The NRL effort was also supported by the USAF Space Test Program and the Office of Naval Research.

The authors also wish to acknowledge the strong support of the STEREO Program Office and others at NASA/GSFC, throughout the project but particularly during the later stages of instrument development and during spacecraft AIT.

\section{REFERENCES}

1. R.A. Howard, J.D. Moses, D.G. Socker and the SECCHI consortium, "Sun Earth connection coronal and heliospheric investigation", SPIE 4139, 2000.

2. B.V. Jackson and C. Leinert, "HELIOS images of solar mass ejections", J. Geophys. Res. 90, 10, 759, 1985.

3. C.J. Eyles, G.M. Simnett, M.P. Cooke, B.V. Jackson, A. Buffington, P.P. Hick, N.R. Waltham, J.M. King, P.A. Anderson and P.E. Holladay, P.E., "The Solar Mass Ejection Imager (SMEI)", Solar Phys. 217, 319, 2003.

4. D.G. Socker, R.A. Howard, C.M. Korendyke, G.M. Simnett and D.F. Webb, "NASA Solar Terrestrial Relations Observatory (STEREO) mission heliospheric imager", SPIE 4139, 2000.

5. R.A. Harrison, C.J. Davis and C.J. Eyles, "The STEREO heliospheric imager: how to detect CMEs in the heliosphere", Adv. Space Research 36, 1512, 2005.

6. J.-M. Defise, J.-P. Halain, E. Mazy, P. Rochus, R.A. Howard, J.D. Moses, D.G. Socker, G.M. Simnett and D.F. Webb, "Design of the Heliospheric Imager for the STEREO mission", SPIE 4498, 2001.

7. J.-M. Defise, J.-P. Halain, E. Mazy, P. Rochus, R.A. Howard, J.D. Moses, D.G. Socker, R.A. Harrison and G.M. Simnett, "Design and tests for the heliospheric imager of the STEREO mission", SPIE 4853, 2003.

8. E. Mazy, J.-P. Halain, J.-M. Defise, P. Ronchain, R.A. Howard, J.D. Moses, C.J. Eyles and R.A. Harrison, "Design and performances of the heliospheric imager for the STEREO mission", SPIE 5962, 2005.

9. S. Koutchmy and P. Lamy, "The F-corona and the circum-solar dust evidences and properties" in "Properties and Interactions of the Interplanetary Dust", IAU Colloq. 85, 63, 1985.

10. e2v technologies datasheet for CCD42-40, http://www.e2v.com.

11. M. Fulle, F. Leblanc, R.A. Harrison, C.J. Davis, C.J. Eyles, J.-P. Halain, R.A. Howard, D. Bockelee-Morvan, G. Cremonese and T. Scarmato, "Discovery of the Atomic Ion Tail of Comet McNaught Using the Heliospheric Imager on STEREO”, Ap. J. Lett. 661, L93, 2007.

12. Astronomy picture of the day 17 January 2007, http://apod.nasa.gov/apod/ap070117.html.

13. R.A. Harrison, C.J. Davis, C.J. Eyles, D. Bewsher, S.R. Crothers, J.A. Davies, R.A. Howard, D.J. Moses, D.G. Socker, J.P.Halain, J.M. Defise, E. Mazy, P. Rochus, D.F. Webb and G.M. Simnett, "First Imaging of Coronal Mass Ejections in the Heliosphere Viewed from Outside the Sun-Earth Line", submitted to Solar Physics, 2007. 